\title{
Operational resource theory of quantum channels
}

\author{
Yunchao Liu $\oplus^{1,2}$ and Xiao Yuan $\oplus^{3, *}$ \\ ${ }^{1}$ Department of Electrical Engineering and Computer Sciences, University of California, Berkeley, California 94720, USA \\ ${ }^{2}$ Center for Quantum Information, Institute for Interdisciplinary Information Sciences, Tsinghua University, Beijing 100084, China \\ ${ }^{3}$ Department of Materials, University of Oxford, Parks Road, Oxford OX1 3PH, United Kingdom
}

(Received 19 May 2019; accepted 7 January 2020; published 11 February 2020)

\begin{abstract}
Quantum resource theories have been widely studied to systematically characterize the nonclassicality of quantum systems. Most resource theories focus on quantum states and study their interconversions. Although quantum channels are generally used as a tool for state manipulation, such a manipulation capability can be naturally regarded as a generalized quantum resource, leading to an open research direction in the resource theories of quantum channels. Various resource-theoretic properties of the channels have been investigated, however, without treating the channels themselves as operational resources that can also be manipulated and converted. In this Rapid Communication, we address this problem by first proposing a general resource framework for quantum channels and introducing resource monotones based on general distance quantifiers of the channels. We study the interplay between the channel and state resource theories by relating the resource monotones of a quantum channel to its manipulation power of the state resource. Regarding channels as operational resources, we introduce asymptotic channel distillation and dilution, the most important tasks in an operational resource theory, and show how to bound the conversion rates with the channel resource monotones. Finally, we apply our results to quantum coherence as an example and introduce the coherence of channels, which characterizes the coherence generation ability of channels. We consider asymptotic channel distillation and dilution with maximally incoherent operations and find the theory asymptotically irreversible, in contrast to the asymptotic reversibility of the coherence of states.
\end{abstract}

DOI: 10.1103/PhysRevResearch.2.012035

Quantum resource theories have been developed as systematic frameworks for the characterization, quantification, and operational interpretation for various quantum effects, including coherence [1-3], discord [4-6], entanglement [7-9], thermodynamics [10,11], magic in stabilizer computation [12-14], etc. Advances in quantum resource theories have not only led to a deeper understanding of the underlying physics, but have also provided new insights and mathematical tools for various quantum information processing tasks that exploit the resources, such as quantum key distribution $[15,16]$, quantum random number generation [17-19], and quantum computing [13,20-24]. We refer to Ref. [25] for a recent review.

A quantum resource theory usually starts by defining three important components: free states, free operations, and resource measures. Free states are those quantum states that do not possess any resource. Free operations are quantum operations that cannot generate resources from free states, and their precise definitions are guided by physical motivations. Resource measures are functionals that map quantum states to real numbers, which cannot be increased under free operations. In an operational resource theory, one of

\footnotetext{
*xiao.yuan.ph@gmail.com

Published by the American Physical Society under the terms of the Creative Commons Attribution 4.0 International license. Further distribution of this work must maintain attribution to the author(s) and the published article's title, journal citation, and DOI.
}

the most important tasks is to study state conversion under free operations. Resource distillation and dilution are optimal schemes that convert between a given state and the maximal resource state, which in general can be uniquely determined in a given resource theory. In many resource theories, such as coherence and entanglement, the distillation and dilution tasks are generally characterized by resource measures based on the relative entropy and $\alpha$-Rényi divergences in the asymptotic independent and identically distributed (i.i.d.) [26-28] and the general one-shot scenarios [29-35], respectively.

Existing developments in quantum resource theories are mainly centered around quantum states, while quantum channels are used as a tool for state resource manipulation. In principle, we can also regard a quantum channel as the resource object and study the resource theory of channels. This has been done mainly for characterizing a certain property of quantum channels, such as channel simulation [36-40], spatial correlations [41], resource generation $[42,43]$, magic quantification $[44,45]$, entanglement of quantum channels [46,47], channel discrimination [48-50], quantum memory [51-53], non-Gaussianity [54], and others [55-58]. Some general conditions that resource theories of channels should satisfy were also formulated [59,60]. Nevertheless, a high-level view of a quantum channel itself as an operational resource has not been well established, partially because the entropic quantifiers for quantum channels have only been developed recently [61-64]. It remains an open direction to study the operational resource theory of quantum channels [25]. 
In this Rapid Communication, we introduce a general framework for the resource theory of quantum channels. We make use of the distance quantifiers of channels, which are defined based on the distance quantifiers of states, to construct resource monotones of the channels. Because the resource theories of states and channels are highly related, we show how the channel resource monotones bound its ability of manipulating state resources. Then we introduce channel distillation and dilution, two fundamental tasks in an operational channel resource theory. By focusing on the resource generation capability of channels, we show how the asymptotic distillation and dilution rates are bounded by the introduced resource monotones. Finally, we take coherence as an example to show the applicability of our general results. We propose the relative entropy of channel coherence and show how it measures the ability of coherence generation. We study channel distillation and dilution with maximally incoherent operations and obtain tight bounds for the asymptotic rates, concluding that the theory is irreversible.

A general resource framework of channels. We first review the resource theory of states. Denote the Hilbert space by $\mathcal{H}$, quantum states by $\mathcal{D}(\mathcal{H})$, and quantum channels by $\mathcal{L}(\mathcal{H})$. A state resource theory in a Hilbert space $\mathcal{H}$ is defined as a tuple $(\Omega, \Phi, \mu)$. Here, $\Omega \subseteq \mathcal{D}(\mathcal{H})$ is the set of free states; $\Phi \subseteq$ $\mathcal{L}(\mathcal{H})$ is the set of free or resource-nongenerating (RNG) operations that preserve free states, i.e., $\phi(\omega) \in \Omega, \forall \phi \in \Phi, \forall \omega \in$ $\Omega$; and $\mu: \mathcal{D}(\mathcal{H}) \rightarrow \mathbb{R}$ are resource measures that satisfy the following:

(S1) Non-negativity: $\mu(\rho) \geqslant 0$ and $\mu(\omega)=0, \forall \omega \in \Omega$;

(S2) Monotonicity: $\mu[\phi(\rho)] \leqslant \mu(\rho), \forall \rho \in \mathcal{D}(\mathcal{H}), \forall \phi \in \Phi$.

When these two properties are satisfied, we called it a resource monotone. Additional requirements of resource measures can be added for a specific resource theory. One popular type of resource measure is based on distance quantifiers $D(\rho, \sigma)$, which satisfy non-negativity $[D(\rho, \sigma) \geqslant 0$ and $D(\rho, \sigma)=0$ if $\rho=\sigma]$ and data-processing inequality $[D[\mathcal{N}(\rho), \mathcal{N}(\sigma)] \leqslant D(\rho, \sigma), \forall \mathcal{N} \in \mathcal{L}(\mathcal{H})]$. Resource quantifiers are defined as the minimal distance to the set of free states, $\mu(\rho)=\min _{\omega \in \Omega} D(\rho, \omega)$, which satisfies (S1) and (S2).

Following a similar mathematical structure, a channel resource theory is also defined as a tuple $(\mathcal{F}, \mathcal{O}, \mathcal{R})$. Free channels $\mathcal{F}$ are those quantum channels that do not have any resource. Free superoperations $\mathcal{O}$ are a subset of superchannels [65] that transforms free channels to free channels. Here, superchannels transform a quantum channel $\mathcal{N}^{A \rightarrow B}$ to another channel $\mathcal{M}^{C \rightarrow D}$ by $\mathcal{M}^{C \rightarrow D}=\mathcal{W}^{B E \rightarrow D} \circ\left(\mathcal{N}^{A \rightarrow B} \otimes \mathrm{id}_{E}\right) \circ$ $\mathcal{V}^{C \rightarrow A E}$, where the superscripts denote input/output systems, id is the identity map, and $\mathcal{W}, \mathcal{V}$ are also quantum channels. The channel resource measures $\mathcal{R}: \mathcal{L}(\mathcal{H}) \rightarrow \mathbb{R}$ map a quantum channel to a real number, satisfying the following:

(R1) Non-negativity: $\mathcal{R}(\mathcal{N}) \geqslant 0 \quad$ and $\quad \mathcal{R}(\mathcal{M})=0$, $\forall \mathcal{M} \in \mathcal{F}$;

(R2) Monotonicity: $\mathcal{R}[\Lambda(\mathcal{N})] \leqslant \mathcal{R}(\mathcal{N}), \forall \mathcal{N} \in \mathcal{L}(\mathcal{H})$, and $\forall \Lambda \in \mathcal{O}$.

We can construct channel resource monotones with distance quantifiers of channels as

$$
\mathcal{R}(\mathcal{N})=\min _{\mathcal{M} \in \mathcal{F}} D(\mathcal{N}, \mathcal{M})
$$

Here, a distance quantifier of two channels $D(\mathcal{N}, \mathcal{M})$ is generally defined by maximizing a distance quantifier of states over all input quantum states [62], $D(\mathcal{N}, \mathcal{M})=$ $\max _{\rho_{A E} \in \mathcal{D}\left(\mathcal{H}_{A E}\right)} D\left[\mathcal{N}_{A} \otimes \operatorname{id}_{E}\left(\rho_{A E}\right), \mathcal{M}_{A} \otimes \operatorname{id}_{E}\left(\rho_{A E}\right)\right]$, where $A$ denotes the system of interest and $E$ is any ancillary system. Examples using this construction include the diamond norm $[66,67]$ and entropies of quantum channels $[61,63,64]$.

Our proposed framework is a natural mathematical extension of state resource theories, which can be used to characterize the general properties of quantum channels. A channel resource theory can be independent of any state resource, such as the quality of a quantum memory [51-53]. However, in common scenarios quantum channels are used to manipulate quantum states, and thus it is natural to further extend our framework where the channel resource theory interacts with a state resource theory. As some channels may generate more resources from the input states than others, we mainly focus on the resource generation ability of the channels. One can also consider the general manipulation abilities of the channels, such as resource detection [60], which we leave to future work.

Interplay with state resource theories. Consider a state resource theory $\mathbf{S}=(\Omega, \Phi, \mu)$ with a tensor product structure, i.e., $\phi \otimes \mathrm{id} \in \Phi, \forall \phi \in \Phi$. To characterize the state resource generating power, we construct a corresponding channel resource theory $\mathbf{C}=(\mathcal{F}, \mathcal{O}, \mathcal{R})$. As RNG channels cannot increase the resource, we define free channels $\mathcal{F}$ to be RNG channels of $\mathbf{S}$. We define free superoperations $\mathcal{O}$ as those quantum superchannels that only use free operations in $\Phi$, i.e., $\Lambda(\mathcal{N})=\phi_{1} \circ(\mathcal{N} \otimes$ id $) \circ \phi_{2}$ for $\phi_{1}, \phi_{2} \in \Phi$. The motivation for this configuration is that since free operations can be applied to manipulate states, they should also be allowed in channel manipulation. Resource monotones can be defined as Eq. (1) with $\mathcal{F}=$ RNG. Alternatively, we introduce a generalized distance quantifier $D_{\Omega}(\mathcal{N}, \mathcal{M})=\max _{\omega_{A E} \in \Omega_{A E}} D\left[\mathcal{N}_{A} \otimes\right.$ $\left.\operatorname{id}_{E}\left(\omega_{A E}\right), \mathcal{M}_{A} \otimes \operatorname{id}_{E}\left(\omega_{A E}\right)\right]$ with a maximization only over free states. Although $D_{\Omega}(\mathcal{N}, \mathcal{M})$ may be increased under a general superchannel, it defines legitimate channel resource monotones,

$$
\mathcal{R}_{\Omega}(\mathcal{N})=\min _{\mathcal{M} \in \mathrm{RNG}} D_{\Omega}(\mathcal{N}, \mathcal{M})
$$

As $D_{\Omega}(\mathcal{N}, \mathcal{M}) \leqslant D(\mathcal{N}, \mathcal{M})$, we have $\mathcal{R}_{\Omega}(\mathcal{N}) \leqslant \mathcal{R}(\mathcal{N})$ for the same distance quantifier $D$. One can further consider an optimization over a general set of states that satisfies certain properties, an interesting open direction of defining general channel resource quantifiers.

Both $\mathcal{R}_{\Omega}(\mathcal{N})$ and $\mathcal{R}(\mathcal{N})$ can be regarded as minimizing distances to free channels. Alternatively, we can also define channel resource monotones by considering the manipulation ability of channels with respect to the resource of quantum states. Based on the intuition that a more powerful channel can generate or boost more resources from input states, we define two channel monotones as

$$
\begin{aligned}
& \mathcal{R}_{g}(\mathcal{N})=\max _{\omega_{A E} \in \Omega_{A E}} \mu\left[\mathcal{N}_{A} \otimes \operatorname{id}_{E}\left(\omega_{A E}\right)\right], \\
& \mathcal{R}_{b}(\mathcal{N})=\max _{\rho_{A E} \in \mathcal{D}\left(\mathcal{H}_{A E}\right)}\left\{\mu\left[\mathcal{N}_{A} \otimes \operatorname{id}_{E}\left(\rho_{A E}\right)\right]-\mu\left(\rho_{A E}\right)\right\},
\end{aligned}
$$

where $\mu(\rho)=\min _{\omega \in \Omega} D(\rho, \omega)$. The resource generating power $\mathcal{R}_{g}(\mathcal{N})$ characterizes the maximal output resource that 
can be generated from free input states, while the resource boosting power $\mathcal{R}_{b}(\mathcal{N})$ characterizes the maximal boosted resource between the output and input states. Similar concepts have been studied in several specific resource theories, including coherence [39,40,68-72], thermodynamics [73], nonGaussianity [54], and others [25]. For a general resource theory, the resource generating/boosting power has been initially proposed by Li et al. [42] with an optimization only over states of system A. Different from Ref. [42], our definitions consider an optimization with ancillae and more general distance measures. We summarize the four types of channel resource monotones constructed above as follows and give the proof in the Supplemental Material [74].

Theorem 1. For any distance quantifier $D(\rho, \sigma)$ satisfying non-negativity and the data-processing inequality, $\mathcal{R}(\mathcal{N}), \mathcal{R}_{\Omega}(\mathcal{N}), \mathcal{R}_{g}(\mathcal{N}), \mathcal{R}_{b}(\mathcal{N})$ are channel resource monotones satisfying (R1) and (R2).

Interestingly, although $\mathcal{R}(\mathcal{N})$ and $\mathcal{R}_{\Omega}(\mathcal{N})$ are defined with channel distance quantifiers, they are closely related to the state resource manipulation power $\mathcal{R}_{g}(\mathcal{N})$ and $\mathcal{R}_{b}(\mathcal{N})$.

Theorem 2. For a state resource theory $\mathbf{S}$ and its corresponding channel resource theory $\mathbf{C}$ as constructed above, we have $\mathcal{R}_{g}(\mathcal{N}) \leqslant \mathcal{R}_{\Omega}(\mathcal{N})$. Furthermore, assuming that $D$ satisfies the triangle inequality, we also have $\mathcal{R}_{b}(\mathcal{N}) \leqslant \mathcal{R}(\mathcal{N})$.

The resource generating power $\mathcal{R}_{g}$ is upper bounded by the generalized distance-based resource monotone $\mathcal{R}_{\Omega}$. For specific resource theories, such as coherence, the equal sign can also be achieved as shown below. Meanwhile, the resource boosting power $\mathcal{R}_{b}(\mathcal{N})$ is also upper bounded by the distancebased monotone $\mathcal{R}(\mathcal{N})$ under certain assumptions. Our results show fundamental connections between the resource theories of quantum channels and states, where the resource manipulation power is upper bounded by distance-based resource monotones, even without specifying the particular resource being studied. Next, we consider the operational tasks of channel distillation and dilution and show their characterizations with the proposed resource monotones.

Channel distillation and dilution. The key to an operational resource theory is to study resource interconversion, where standard operational tasks include resource distillation and dilution. For a channel resource theory $\mathbf{C}=(\mathcal{F}, \mathcal{O}, \mathcal{R})$, we assume that there exists a set of optimal unit resource channels $\mathfrak{G}$ which are defined from physical considerations. Then a natural question is how can we convert nonoptimal channels to optimal ones and vice versa. For a channel resource theory constructed from a state resource theory, a natural definition of the optimal unit resource channels are those that have a maximal resource generating power, i.e., those quantum channels that can generate a maximal unit resource state from certain free states. For example, the Hadamard gate has a maximal resource generating power in the resource theory of coherence. There also exist channel resource theories that do not rely on state resources, including quantum memory [51-53] and channel purity, in which unitary channels are regarded as optimal resources. With the definition of optimal unit resource channels, we define asymptotic quantum channel distillation/dilution based on two ways of using the channels.
Definition 1. The parallel quantum channel distillation/dilution rate is defined as

$$
\begin{aligned}
\mathcal{R}_{\text {distill }}(\mathcal{N})= & \lim _{\varepsilon \rightarrow 0^{+}} \lim _{n \rightarrow \infty} \max \{R: \exists \mathcal{G} \in \mathfrak{G}, \Lambda \in \mathcal{O}, \\
& \left.\left\|\Lambda\left(\mathcal{N}^{\otimes n}\right)-\mathcal{G}^{\otimes n R}\right\|_{\diamond} \leqslant \varepsilon\right\}, \\
\mathcal{R}_{\text {dilute }}(\mathcal{N})= & \lim _{\varepsilon \rightarrow 0^{+}} \lim _{n \rightarrow \infty} \min \{R: \exists \mathcal{G} \in \mathfrak{G}, \Lambda \in \mathcal{O}, \\
& \left.\left\|\Lambda\left(\mathcal{G}^{\otimes n R}\right)-\mathcal{N}^{\otimes n}\right\|_{\diamond} \leqslant \varepsilon\right\} .
\end{aligned}
$$

Definition 2. The iterative quantum channel distillation/dilution rate is defined as

$$
\begin{aligned}
\tilde{\mathcal{R}}_{\text {distill }}(\mathcal{N})= & \lim _{\varepsilon \rightarrow 0^{+}} \lim _{n \rightarrow \infty} \max \left\{R: \exists \mathcal{G} \in \mathfrak{G}, \Lambda_{i} \in \mathcal{O},\right. \\
& \left.\left\|\Lambda_{1}(\mathcal{N}) \circ \cdots \circ \Lambda_{n}(\mathcal{N})-\mathcal{G}^{\otimes n R}\right\|_{\diamond} \leqslant \varepsilon\right\}, \\
\tilde{\mathcal{R}}_{\text {dilute }}(\mathcal{N})= & \lim _{\varepsilon \rightarrow 0^{+}} \lim _{n \rightarrow \infty} \min \left\{R: \exists \mathcal{G} \in \mathfrak{G}, \Lambda_{i} \in \mathcal{O},\right. \\
& \left.\left\|\Lambda_{1}(\mathcal{G}) \circ \cdots \circ \Lambda_{n R}(\mathcal{G})-\mathcal{N}^{\otimes n}\right\|_{\diamond} \leqslant \varepsilon\right\} .
\end{aligned}
$$

Here, $\left\|\mathcal{N}_{A}\right\|_{\diamond}=\max _{\rho_{A E} \in \mathcal{D}\left(\mathcal{H}_{A E}\right)} \operatorname{Tr}\left|\mathcal{N}_{A} \otimes \operatorname{id}_{E}\left(\rho_{A E}\right)\right|$ denotes the diamond norm of channels. Note that the iterative protocols are stronger than the parallel ones as the channels are used independently so that the outputs of the channels can further be inputs of other channels. Therefore we have $\mathcal{R}_{\text {distill }}(\mathcal{N}) \leqslant$ $\tilde{\mathcal{R}}_{\text {distill }}(\mathcal{N}) \leqslant \tilde{\mathcal{R}}_{\text {dilute }}(\mathcal{N}) \leqslant \mathcal{R}_{\text {dilute }}(\mathcal{N})$. However, the distilled or diluted channels after the iterative protocols can only be used in parallel, as their input spaces are in a tensor product. If we want to obtain independent channels that can be used iteratively, we can distill the channels one by one. Such a scenario corresponds to one-shot channel distillation and dilution, which will be considered in future work.

Next, we show how resource monotones are related to the asymptotic distillation/dilution rate. For this purpose, we introduce asymptotic resource measures, a universal set of channel resource measures which are useful for characterizing asymptotic operational tasks. They are defined as resource monotones that satisfy further requirements besides (R1) and (R2), including the following:

(R3) Normalization: $\mathcal{R}(\mathcal{G})=1$ for all $\mathcal{G} \in \mathfrak{G}$;

(R4) Additivity: $\mathcal{R}(\mathcal{N} \otimes \mathcal{M})=\mathcal{R}(\mathcal{N})+\mathcal{R}(\mathcal{M})$;

(R4') Subadditivity: $\mathcal{R}(\mathcal{N} \otimes \mathcal{M}) \leqslant \mathcal{R}(\mathcal{N})+\mathcal{R}(\mathcal{M})$;

(R5) Continuity: $|\mathcal{R}(\mathcal{N})-\mathcal{R}(\mathcal{M})| \leqslant O(f(\varepsilon) \log d)$ with $\lim _{\varepsilon \rightarrow 0^{+}} f(\varepsilon)=0$, when $\|\mathcal{N}-\mathcal{M}\|_{\diamond} \leqslant \varepsilon$.

Here, $d$ denotes the dimension of the Hilbert space of the outputs. We note that $\left(\mathrm{R} 4^{\prime}\right)$ is a weaker version of (R4). The asymptotic resource measures play an important role in parallel channel distillation/dilution.

Theorem 3. For any asymptotic resource measure $\mathcal{R}_{\text {asymp }}(\mathcal{N})$ satisfying (R1)-(R5), we have

$$
\mathcal{R}_{\text {distill }}(\mathcal{N}) \leqslant \mathcal{R}_{\text {asymp }}(\mathcal{N}) \leqslant \mathcal{R}_{\text {dilute }}(\mathcal{N}) .
$$

It is in general hard to explicitly determine the distillation/dilution rate as well as constructing asymptotic resource measures without specifying the resource structure. However, following the spirit of studying the resource generating power of quantum channels, we can focus on the special case where the optimal unit resource channel is uniquely defined to be the channel $\mathcal{G}_{\star}(\rho)=\rho_{m}$, which constantly outputs the maximal unit resource state $\rho_{m}$ and is the strongest channel for generating state resources. This definition aligns 
with the intuition that channels which generate more powerful resource states are more resourceful. In this case, we are able to characterize the distillation and dilution rates with the resource generating/boosting power of channels in the general setting, which can be applied to all channel resource theories with a corresponding state resource.

Assuming that the state resource monotone $\mu$ satisfies similar requirements as (R1)-(R5), we show that the distillation rates can be explicitly determined.

Theorem 4. When $\mathfrak{G}=\left\{\mathcal{G}_{\star}\right\}$, the parallel and iterative channel distillation rates satisfy

$$
\begin{aligned}
\mathcal{R}_{g}(\mathcal{N}) & \leqslant \mathcal{R}_{\text {distill }}(\mathcal{N}) \leqslant \lim _{n \rightarrow \infty} \frac{1}{n} \mathcal{R}_{g}\left(\mathcal{N}^{\otimes n}\right), \\
\tilde{\mathcal{R}}_{\text {distill }}(\mathcal{N}) & \leqslant \mathcal{R}_{b}(\mathcal{N}),
\end{aligned}
$$

where we assume that $\mathcal{R}_{g}$ satisfies (R3) and (R5) and $\mathcal{R}_{b}$ satisfies (R3). The equal sign $\mathcal{R}_{g}(\mathcal{N})=\mathcal{R}_{\text {distill }}(\mathcal{N})$ is achieved if $\mathcal{R}_{g}$ also satisfies $\left(\mathrm{R} 4^{\prime}\right)$, and $\tilde{\mathcal{R}}_{\text {distill }}(\mathcal{N})=\mathcal{R}_{b}(\mathcal{N})$ is achieved when the state resource theory is asymptotically reversible.

Our results verify the intuition that the resource generating/boosting power quantifies how much resource a channel can generate/boost in the asymptotic operational setting. Furthermore, they indicate a possible separation between iterative and parallel protocols in the distillation task, as $\mathcal{R}_{b}$ is generally larger than $\mathcal{R}_{g}$. Meanwhile, we find that the parallel and iterative dilution protocols are actually equivalent, and lower bounded by $\mathcal{R}_{b}(\mathcal{N})$.

Theorem 5. When $\mathfrak{G}=\left\{\mathcal{G}_{\star}\right\}$, the parallel and iterative channel dilution rates satisfy

$$
\mathcal{R}_{\text {dilute }}(\mathcal{N})=\tilde{\mathcal{R}}_{\text {dilute }}(\mathcal{N}) \geqslant \mathcal{R}_{b}(\mathcal{N})
$$

where we assume that $\mathcal{R}_{b}(\mathcal{N})$ satisfies (R3) and (R5).

Note that the channel dilution protocol is closely related to channel simulation using a state resource, which has been studied for various resource theories [25]. In general, one can also study other manipulation powers of the channels, where different (maybe also nonequivalent) optimal resource channels can be considered for different purposes. We leave the general case for future work as this Rapid Communication aims to establish the basic resource framework of the channels and study its interplay with state resource theories.

Following the quantification of the asymptotic rates, a particular interesting property to study for an operational resource theory is the asymptotic reversibility, which exists in both the resource theories of entanglement and coherence [32,75-77]. In the context of channel resource theory, asymptotic reversibility is defined as $\mathcal{R}_{\text {distill }}(\mathcal{N})=\mathcal{R}_{\text {dilute }}(\mathcal{N})$ for all quantum channels $\mathcal{N}$. It is unclear whether a reversible channel resource theory exists without specifying the resource structure. When focusing on resource generation, our results indicate that the channel resource theory is irreversible as long as there exists a channel $\mathcal{N}$ such that $\mathcal{R}_{b}(\mathcal{N})>$ $\lim _{n \rightarrow \infty} \frac{1}{n} \mathcal{R}_{g}\left(\mathcal{N}^{\otimes n}\right)$. While our results contribute as an initial step, establishing the reversibility criteria for general channel resource theories requires extensive studies on the asymptotic behavior of channel resource quantifiers [77,78]. In the following, we give an explicit example in the context of quantum coherence. We show that even though the state resource theory of coherence is reversible, the corresponding channel resource theory is not.

Coherence. We first review the resource theory of coherence. For a fixed basis $\{|i\rangle\}_{i=0}^{d-1}$ in a $d$-dimensional Hilbert space, free states or incoherent states are those diagonal in the basis, i.e., $\left.\mathcal{I}=\left\{\delta\left|\delta=\sum_{i=0}^{d-1} \delta_{i}\right| i\right\rangle\langle i|\right\}$. Meanwhile, maximal resource states are those with a uniform superposition of basis states. For free operations, we consider the maximal set of operations, maximal incoherent operations (MIOs) [1], which map incoherent states to incoherent states. Interestingly, the resource theory of coherence is asymptotically reversible under MIOs [32], characterized by the relative entropy of coherence $C_{r}(\rho)=\min _{\delta \in \mathcal{I}} S(\rho \| \delta)$. Here, $S(\rho \| \sigma)=\operatorname{Tr}(\rho \log \rho)-$ $\operatorname{Tr}(\rho \log \sigma)$ is the quantum relative entropy.

Now we construct a channel resource theory of coherence to characterize the coherence generating power. This has been partially done in several works [39,40,60,68,70-72], whereas they did not treat channel coherence as an operational resource. Following our resource framework, we define free channels as resource nongenerating channels, i.e., MIOs. Free superoperations are a subset of superchannels that transform a quantum channel into another using MIOs, $\mathcal{N} \rightarrow \mathcal{M}_{1} \circ$ $(\mathcal{N} \otimes$ id $) \circ \mathcal{M}_{2}$ for all $\mathcal{M}_{1}, \mathcal{M}_{2} \in$ MIO. Finally, following our general results, we have four resource monotones,

$$
\begin{aligned}
\mathcal{C}_{r}(\mathcal{N}) & =\min _{\mathcal{M} \in \mathrm{MIO}} \max _{\rho \in \mathcal{D}\left(\mathcal{H}_{A E}\right)} S[\mathcal{N} \otimes \operatorname{id}(\rho) \| \mathcal{M} \otimes \operatorname{id}(\rho)], \\
\mathcal{C}_{r, \mathcal{I}}(\mathcal{N}) & =\min _{\mathcal{M} \in \mathrm{MIO}} \max _{\delta \in \mathcal{I}_{A E}} S[\mathcal{N} \otimes \operatorname{id}(\delta) \| \mathcal{M} \otimes \operatorname{id}(\delta)], \\
\mathcal{C}_{r, g}(\mathcal{N}) & =\max _{\delta \in \mathcal{I}_{A E}} C_{r}[\mathcal{N} \otimes \operatorname{id}(\delta)] \\
\mathcal{C}_{r, b}(\mathcal{N}) & =\max _{\rho \in \mathcal{D}\left(\mathcal{H}_{A E}\right)}\left\{C_{r}[\mathcal{N} \otimes \operatorname{id}(\rho)]-C_{r}(\rho)\right\}
\end{aligned}
$$

To further study these four monotones, we utilize the resource structure of coherence, including that it has a resource destroying map [79]. Generalizing Theorem 2, we show that the channel resource monotone $\mathcal{C}_{r, \mathcal{I}}(\mathcal{N})$ is actually equal to the resource generating power $\mathcal{C}_{r, g}(\mathcal{N})$,

$$
\mathcal{C}_{r, \mathcal{I}}(\mathcal{N})=\mathcal{C}_{r, g}(\mathcal{N})
$$

Furthermore, using the convexity of $C_{r}$, we have $\mathcal{C}_{r, \mathcal{I}}(\mathcal{N})=$ $\max _{i} C_{r}[\mathcal{N}(|i\rangle\langle i|)]$, showing that $\mathcal{C}_{r, \mathcal{I}}(\mathcal{N})$ is efficiently computable. This simplified expression allows us to prove many useful properties and conclude that $\mathcal{C}_{r, \mathcal{I}}(\mathcal{N})$ is actually an asymptotic resource measure satisfying (R1)-(5). By definition, we have $\mathcal{C}_{r, \mathcal{I}}(\mathcal{N}) \leqslant \mathcal{C}_{r}(\mathcal{N})$ and $\mathcal{C}_{r, g}(\mathcal{N}) \leqslant \mathcal{C}_{r, b}(\mathcal{N})$. However, as the relative entropy violates the triangle inequality, we cannot decide the relation between $\mathcal{C}_{r}(\mathcal{N})$ and $\mathcal{C}_{r, b}(\mathcal{N})$.

Considering channel distillation/dilution, we define optimal unit resource channels as $\mathfrak{G}=\left\{\mathcal{G}_{\star} \mid \mathcal{G}_{\star}(\rho)=\Psi_{2}\right\}$ with the maximal coherent qubit state $\left|\Psi_{2}\right\rangle=(|0\rangle+|1\rangle) / \sqrt{2}$. As shown in Ref. [40], such a channel can simulate an arbitrary channel that outputs a qubit. Denote the asymptotic parallel (iterative) channel coherence distillation/dilution rate as $\mathcal{C}_{\text {distill }}$ $\left(\tilde{\mathcal{C}}_{\text {distill }}\right)$ and $\mathcal{C}_{\text {dilute }}\left(\tilde{\mathcal{C}}_{\text {dilute }}\right)$, respectively. As an immediate consequence of Theorems 3 and 4 , it follows that $\mathcal{C}_{\text {distill }}(\mathcal{N}) \leqslant$ $\mathcal{C}_{r, \mathcal{I}}(\mathcal{N}) \leqslant \mathcal{C}_{\text {dilute }}(\mathcal{N})$ and $\tilde{\mathcal{C}}_{\text {distill }}(\mathcal{N})=\mathcal{C}_{r, b}(\mathcal{N})$. For parallel distillation, we are also able to show that the equal sign is achieved, due to the additivity of $\mathcal{C}_{r, \mathcal{I}}(\mathcal{N})$. 
Corollary 1 . The parallel and iterative asymptotic distillation rates of channel coherence are $\mathcal{C}_{\text {distill }}(\mathcal{N})=\mathcal{C}_{r, \mathcal{I}}(\mathcal{N})$ and $\tilde{\mathcal{C}}_{\text {distill }}(\mathcal{N})=\mathcal{C}_{r, b}(\mathcal{N})$, respectively.

Note that there exists a quantum channel $\mathcal{N}$ such that $\mathcal{C}_{r, b}(\mathcal{N})>\mathcal{C}_{r, \mathcal{I}}(\mathcal{N})$ (see the Supplemental Material [74] for an explicit example) and therefore $\mathcal{C}_{\text {dilute }}(\mathcal{N})=\tilde{\mathcal{C}_{\text {dilute }}}(\mathcal{N}) \geqslant$ $\tilde{\mathcal{C}}_{\text {distill }}(\mathcal{N})>\mathcal{C}_{\text {distill }}(\mathcal{N})$, indicating the irreversibility of the operational resource theory of channel coherence.

Corollary 2. The channel resource theory of coherence is asymptotically irreversible.

For the dilution part, besides the lower bound given in Theorem 5, we consider the (smooth) maximum entropy of channel coherence,

$$
\begin{aligned}
\mathcal{C}_{\max }^{\varepsilon}(\mathcal{N}) & =\min _{\left\|\mathcal{N}-\mathcal{N}^{\prime}\right\|_{0} \leqslant \varepsilon} \log \min \{\lambda: \exists \mathcal{M} \in \mathrm{MIO}, \\
\mathcal{N}^{\prime} & \leqslant \lambda \mathcal{M}\},
\end{aligned}
$$

which is used to characterize one-shot channel simulation using coherence [40]. We show that the asymptotic dilution rate is equal to its regularized version.

Theorem 6 . The asymptotic dilution rate of channel coherence is equal to the regularized maximum entropy of channel coherence,

$$
\mathcal{C}_{\text {dilute }}(\mathcal{N})=\tilde{\mathcal{C}}_{\text {dilute }}(\mathcal{N})=\mathcal{C}_{\text {max }}^{\infty}(\mathcal{N}),
$$

where $\mathcal{C}_{\max }^{\infty}(\mathcal{N})=\lim _{\varepsilon \rightarrow 0^{+}} \lim _{n \rightarrow \infty} \frac{1}{n} \mathcal{C}_{\max }^{\varepsilon}\left(\mathcal{N}^{\otimes n}\right)$.

Calculating the limit requires the development of a channel analogy of the generalized quantum Stein's lemma [80], which is beyond the scope of this Rapid Communication and is left as important future work.

Discussion. Our work introduces a general framework for the resource theory of quantum channels and study of its interplay with the resource theory of states. We also introduce the operational tasks of channel distillation and dilution and study their characterizations with channel resource measures. We consider coherence as an example and find the channel resource theory of coherence asymptotically irreversible. Future work can extend our results to other quantum resources that have a specific resource structure. As a higher-level resource, channel resource theory can focus on the manipulation power of state resources, including coherence [39,40], entanglement [47], discord, magic [44,45], and thermodynamics. More interestingly, channel resources can be independent of state resources, such as quantum memory [51-53] and channel purity. Meanwhile, previous works have mainly focused on certain properties of the channels without considering quantum channels as operational resources. Completing the operational resource framework of channels requires extensive future work, such as studying the basic entropic quantifiers of the channels [61-64] and investigating channel conversions [81]. We hope our work establishes the basic framework and can inspire future studies of channel resource theories.

Note added. Recently, we became aware of a closely related work by Liu and Winter [82], who independently proposed a similar framework for channel resource theories and further studied the robustness measure and its role in resource erasure.

Acknowledgments. We acknowledge Qi Zhao for insightful discussions. Y.L. was supported by the National Natural Science Foundation of China Grants No. 11875173 and No. 11674193, and the National Key R\&D Program of China Grants No. 2017YFA0303900 and No. 2017YFA0304004. X.Y. was supported by the EPSRC National Quantum Technology Hub in Networked Quantum Information Technology (EP/M013243/1).
[1] J. Aberg, arXiv:quant-ph/0612146.

[2] T. Baumgratz, M. Cramer, and M. B. Plenio, Phys. Rev. Lett. 113, 140401 (2014)

[3] A. Streltsov, G. Adesso, and M. B. Plenio, Rev. Mod. Phys. 89, 041003 (2017).

[4] L. Henderson and V. Vedral, J. Phys. A: Math. Gen. 34, 6899 (2001).

[5] H. Ollivier and W. H. Zurek, Phys. Rev. Lett. 88, 017901 (2001).

[6] K. Modi, A. Brodutch, H. Cable, T. Paterek, and V. Vedral, Rev. Mod. Phys. 84, 1655 (2012).

[7] C. H. Bennett, H. J. Bernstein, S. Popescu, and B. Schumacher, Phys. Rev. A 53, 2046 (1996).

[8] V. Vedral, M. B. Plenio, M. A. Rippin, and P. L. Knight, Phys. Rev. Lett. 78, 2275 (1997).

[9] R. Horodecki, P. Horodecki, M. Horodecki, and K. Horodecki, Rev. Mod. Phys. 81, 865 (2009).

[10] F. G. S. L. Brandão, M. Horodecki, J. Oppenheim, J. M. Renes, and R. W. Spekkens, Phys. Rev. Lett. 111, 250404 (2013).

[11] J. Goold, M. Huber, A. Riera, L. del Rio, and P. Skrzypczyk, J. Phys. A: Math. Theor. 49, 143001 (2016).

[12] V. Veitch, C. Ferrie, D. Gross, and J. Emerson, New J. Phys. 14, 113011 (2012).
[13] V. Veitch, S. H. Mousavian, D. Gottesman, and J. Emerson, New J. Phys. 16, 013009 (2014).

[14] M. Howard and E. Campbell, Phys. Rev. Lett. 118, 090501 (2017).

[15] I. Devetak and A. Winter, Proc. R. Soc. A 461, 207 (2005).

[16] P. J. Coles, E. M. Metodiev, and N. Lütkenhaus, Nat. Commun. 7, 11712 (2016).

[17] X. Yuan, H. Zhou, Z. Cao, and X. Ma, Phys. Rev. A 92, 022124 (2015).

[18] X. Yuan, Q. Zhao, D. Girolami, and X. Ma, Adv. Quantum Technol. 2, 1900053 (2019).

[19] M. Hayashi and H. Zhu, Phys. Rev. A 97, 012302 (2018).

[20] N. Datta, IEEE Trans. Inf. Theory 55, 2816 (2009).

[21] S. Bravyi, G. Smith, and J. A. Smolin, Phys. Rev. X 6, 021043 (2016).

[22] M. Howard, J. Wallman, V. Veitch, and J. Emerson, Nature (London) 510, 351 (2014).

[23] M. Hillery, Phys. Rev. A 93, 012111 (2016).

[24] N. Anand and A. K. Pati, arXiv:1611.04542.

[25] E. Chitambar and G. Gour, Rev. Mod. Phys. 91, 025001 (2019).

[26] E. M. Rains, IEEE Trans. Inf. Theory 47, 2921 (2001).

[27] P. M. Hayden, M. Horodecki, and B. M. Terhal, J. Phys. A: Math. Gen. 34, 6891 (2001). 
[28] A. Winter and D. Yang, Phys. Rev. Lett. 116, 120404 (2016).

[29] F. Buscemi and N. Datta, J. Math. Phys. 51, 102201 (2010).

[30] F. G. S. L. Brandao and N. Datta, IEEE Trans. Inf. Theory 57, 1754 (2011).

[31] F. Buscemi and N. Datta, Phys. Rev. Lett. 106, 130503 (2011).

[32] Q. Zhao, Y. Liu, X. Yuan, E. Chitambar, and X. Ma, Phys. Rev. Lett. 120, 070403 (2018).

[33] B. Regula, K. Fang, X. Wang, and G. Adesso, Phys. Rev. Lett. 121, 010401 (2018).

[34] Q. Zhao, Y. Liu, X. Yuan, E. Chitambar, and A. Winter, IEEE Trans. Inf. Theory 65, 6441 (2019).

[35] Z.-W. Liu, K. Bu, and R. Takagi, Phys. Rev. Lett. 123, 020401 (2019).

[36] M. Berta, F. G. S. L. Brandão, M. Christandl, and S. Wehner, IEEE Trans. Inf. Theory 59, 6779 (2013).

[37] S. Pirandola, R. Laurenza, C. Ottaviani, and L. Banchi, Nat. Commun. 8, 15043 (2017).

[38] M. M. Wilde, Phys. Rev. A 98, 042338 (2018).

[39] K. Ben Dana, M. García Díaz, M. Mejatty, and A. Winter, Phys. Rev. A 95, 062327 (2017).

[40] M. G. Díaz, K. Fang, X. Wang, M. Rosati, M. Skotiniotis, J. Calsamiglia, and A. Winter, Quantum 2, 100 (2018).

[41] Á. Rivas and M. Müller, New J. Phys. 17, 062001 (2015).

[42] L. Li, K. Bu, and Z.-W. Liu, arXiv:1812.02572.

[43] R. Takagi and B. Regula, Phys. Rev. X 9, 031053 (2019).

[44] J. R. Seddon and E. Campbell, Proc. R. Soc. A 475, 20190251 (2019).

[45] X. Wang, M. M. Wilde, and Y. Su, New J. Phys. 21, 103002 (2019).

[46] C. H. Bennett, A. W. Harrow, D. W. Leung, and J. A. Smolin, IEEE Trans. Inf. Theory 49, 1895 (2003).

[47] E. Kaur and M. M. Wilde, J. Phys. A: Math. Theor. 51, 035303 (2017).

[48] S. Pirandola and C. Lupo, Phys. Rev. Lett. 118, 100502 (2017).

[49] M. Berta, C. Hirche, E. Kaur, and M. M. Wilde, arXiv:1808.01498.

[50] S. Pirandola, R. Laurenza, C. Lupo, and J. L. Pereira, npj Quantum Inf. 5, 50 (2019).

[51] D. Rosset, F. Buscemi, and Y.-C. Liang, Phys. Rev. X 8, 021033 (2018).

[52] T. Simnacher, N. Wyderka, C. Spee, X.-D. Yu, and O. Gühne, Phys. Rev. A 99, 062319 (2019).

[53] X. Yuan, Y. Liu, Q. Zhao, B. Regula, J. Thompson, and M. Gu, arXiv:1907.02521.

[54] Q. Zhuang, P. W. Shor, and J. H. Shapiro, Phys. Rev. A 97, 052317 (2018).

[55] J.-H. Hsieh, S.-H. Chen, and C.-M. Li, Sci. Rep. 7, 13588 (2017).
[56] S.-H. Chen, H. Lu, Q.-C. Sun, Q. Zhang, Y.-A. Chen, and C.-M. Li, Phys. Rev. Research 2, 013043 (2020).

[57] C.-C. Kuo, S.-H. Chen, W.-T. Lee, H.-M. Chen, H. Lu, and C.-M. Li, Sci. Rep. 9, 20316 (2019).

[58] E. Wolfe, D. Schmid, A. B. Sainz, R. Kunjwal, and R. W. Spekkens, arXiv:1903.06311.

[59] B. Coecke, T. Fritz, and R. W. Spekkens, Inf. Comput. 250, 59 (2016).

[60] T. Theurer, D. Egloff, L. Zhang, and M. B. Plenio, Phys. Rev. Lett. 122, 190405 (2019).

[61] T. Cooney, M. Mosonyi, and M. M. Wilde, Commun. Math. Phys. 344, 797 (2016).

[62] F. Leditzky, E. Kaur, N. Datta, and M. M. Wilde, Phys. Rev. A 97, 012332 (2018).

[63] X. Yuan, Phys. Rev. A 99, 032317 (2019)

[64] G. Gour and M. M. Wilde, arXiv:1808.06980.

[65] G. Chiribella, G. M. D'Ariano, and P. Perinotti, Europhys. Lett. 83, 30004 (2008).

[66] D. Aharonov, A. Kitaev, and N. Nisan, in Proceedings of the Thirtieth Annual ACM Symposium on Theory of Computing, STOC '98 (ACM, New York, 1998), pp. 20-30.

[67] J. Watrous, Theory Comput. 5, 217 (2009).

[68] A. Mani and V. Karimipour, Phys. Rev. A 92, 032331 (2015).

[69] M. García-Díaz, D. Egloff, and M. B. Plenio, Quantum Inf. Comput. 16, 1282 (2016).

[70] P. Zanardi, G. Styliaris, and L. Campos Venuti, Phys. Rev. A 95, 052307 (2017).

[71] P. Zanardi, G. Styliaris, and L. Campos Venuti, Phys. Rev. A 95, 052306 (2017)

[72] K. Bu, A. Kumar, L. Zhang, and J. Wu, Phys. Lett. A 381, 1670 (2017).

[73] M. Navascués and L. P. García-Pintos, Phys. Rev. Lett. 115, 010405 (2015).

[74] See Supplemental Material at http://link.aps.org/supplemental/ 10.1103/PhysRevResearch.2.012035 for detailed proofs.

[75] F. G. Brandao and M. B. Plenio, Nat. Phys. 4, 873 (2008).

[76] F. G. S. L. Brandão and M. B. Plenio, Commun. Math. Phys. 295, 829 (2010).

[77] F. G. S. L. Brandão and G. Gour, Phys. Rev. Lett. 115, 070503 (2015).

[78] G. Gour and A. Winter, Phys. Rev. Lett. 123, 150401 (2019).

[79] Z.-W. Liu, X. Hu, and S. Lloyd, Phys. Rev. Lett. 118, 060502 (2017).

[80] F. G. S. L. Brandão and M. B. Plenio, Commun. Math. Phys. 295, 791 (2010).

[81] G. Gour, IEEE Trans. Inf. Theory 65, 5880 (2019).

[82] Z.-W. Liu and A. Winter, arXiv:1904.04201. 